Mots. Les langages du politique

\title{
L'adjectif berlusconien dans la presse française. Une illustration de l'emploi métaphorique d'un dérivé du nom propre en discours
}

The adjective Berlusconien in the French press. An example of the metaphorical use of an adjective derived from a proper noun El adjectivo berlusconien en la prensa francesa. Una ilustración del empleo metafórico de un derivado del nombre propio en el discurso

Paola Paissa

\section{OpenEdition}

Journals

Édition électronique

URL : https://journals.openedition.org/mots/22277

DOI : $10.4000 /$ mots. 22277

ISSN : 1960-6001

Éditeur

ENS Éditions

Édition imprimée

Date de publication : 9 mai 2016

Pagination : 155-170

ISBN : 978-2-84788-793-8

ISSN : 0243-6450

\section{Référence électronique}

Paola Paissa, «L'adjectif berlusconien dans la presse française. Une illustration de l'emploi métaphorique d'un dérivé du nom propre en discours », Mots. Les langages du politique [En ligne], 110 | 2016, mis en ligne le 09 mai 2018, consulté le 24 avril 2022. URL : http://journals.openedition.org/ mots/22277 ; DOI : https://doi.org/10.4000/mots.22277 


\section{L'adjectif berlusconien dans la presse française. Une illustration de l'emploi métaphorique d'un dérivé du nom propre en discours}

Les formations dérivées du nom propre d'hommes politiques font l'objet d'une grande productivité dans le langage médiatique, donnant lieu à des néologismes variés et souvent éphémères (ex. : raffarinade, juppéiser, mégrétiser, etc.). Dans cet ensemble hétéroclite et mouvant, les adjectifs déonomastiques suffixés en -ien, comme lepenien, sarkozyen, sont tout particulièrement fréquents. Utilisés en principe en tant qu'adjectifs relationnels, c'est-à-dire susceptibles de remplacer le complément du nom (ex. : une mesure sarkozyenne $\rightarrow$ une mesure de Sarkozy), ou comme qualificatifs, éventuellement à « emploi partisan ", ces adjectifs peuvent connaître, bien que plus rarement, un usage figural $^{1}$. Cet emploi se vérifie lorsque ces unités ne réfèrent plus au porteur habituel du nom propre dont elles dérivent, s’appliquant, en revanche, à un référent autre, sur lequel elles transfèrent un certain nombre de propriétés que la doxa interdiscursive associe normalement au nom propre dont ces dérivés sont issus. L'exemple ci-dessous, concernant l'adjectif poutinien, illustre bien ce dispositif :

1. Les détracteurs du Premier ministre [Erdogan] lui reprochent un exercice «poutinien » du pouvoir. (Le Nouvel Observateur, 7 juin 2013)

Dans cet énoncé, assez trivial dans la communication médiatique, plusieurs mécanismes langagiers d'ordre différent sont mis en œuvre. Sur le plan énonciatif, cette assertion, exprimant le point de vue (Rabatel, 2005) des « détracteurs » d'Erdogan, fonctionne sur un mode allusif (Moirand, 2007), accordant une marge interprétative très vaste au destinataire, convié à une coopération

1. Pour l'alternance des valeurs relationnelles et qualificatives d'un adjectif dérivé du nom propre, voir Bartning et Noailly, 1993. L'analyse conduite sur bolivariano dans Samouth, 2010, se fonde entièrement sur cette alternance. Par ailleurs, le suffixe adjectival -ien se charge de plusieurs fonctions, remplaçant, dans certains cas, le suffixe -iste dans l'indication de l'appartenance politique, ou «emploi partisan» (Lignon, 2002). Ces études ne prennent jamais en compte, en revanche, l'usage figural qui nous occupe ici. 
tout particulièrement active. Sur le plan logico-argumentatif, la proposition aboutit à un amalgame discursif reposant sur une relation analogique (Koren, 2012) grâce à laquelle on transpose sur Erdogan (ou, plus précisément, sur son "exercice du pouvoir») un faisceau de traits sémantiques sous lesquels une certaine communauté de locuteurs catégorise le nom propre Poutine.

Suivant une hypothèse que nous nous proposons d'approfondir ailleurs sur le plan théorique, la qualification réalisée par l'adjectif déonomastique a ainsi partie liée avec le phénomène qu'une vaste bibliographie définit comme l'«emploi métaphorique » du nom propre2 (Jonasson, 1991; Gary-Prieur, 1994 ; Kleiber, 1995), emploi dont relève, par exemple, l'énoncé suivant :

2. Viktor Orbàn, le petit Poutine de Budapest. (L'Express, 21 février 2015)

En effet, le déonomastique adjectival de l'exemple 1 (poutinien) véhicule le même ensemble de valeurs sémantiques et paradigmatiques que celles qui sont discursivement attachées au nom propre Poutine, ensemble que l'adjectif transfère sur un autre référent (Erdogan). Autrement dit, en 1, on interprète poutinien au travers du nom propre Poutine, qui circule dans l'interdiscours en tant que parangon, ou «meilleur représentant», du chef autoritaire, despotique, machiste, nationaliste, manipulateur des lois, etc., comme l'illustre efficacement l'exemple 2, où ces valeurs sont reportées, par un vecteur métaphorique, sur Viktor Orbàn. En relation à cette matrice figurale, que nous supposons être commune au nom propre et à son dérivé, nous parlerons ici d' «emploi métaphorique » de l'adjectif déonomastique. Ce type d'emploi a été jusqu'ici fort peu étudié, à notre connaissance, pour ce qui concerne les formations construites morphologiquement à partir du nom propre, notamment dans la perspective sémantico-discursive qui est la nôtre3.

Dans ce qui suit, nous nous proposons donc de déceler et décrire l'emploi métaphorique de l'adjectif berlusconien, que nous avons choisi en tant qu'angle d'observation du comportement discursif des dérivés adjectivaux du nom propre. Nous appuyant sur un corpus de presse française, notre objectif est de rendre compte de certains effets de généralisation et d'équivalence trompeuse, caractéristiques du discours de presse, que permet et favorise le dispositif rhétorique formant l'objet de notre étude. Cependant, avant d'entamer notre analyse, quelques précisions s’imposent concernant la terminologie à laquelle nous aurons recours, ainsi que la nature et la constitution de notre corpus.

2. Cet emploi est également décrit sous l'étiquette d'« antonomase » du nom propre (Flaux, 1991, 2000 ; Leroy, 2000, 2004, 2005b). Sans entrer dans des questions terminologiques subtiles (voir Kleiber, 1994), nous préférons utiliser ici la dénomination d' "emploi métaphorique » du nom propre, à la fois plus consensuelle et plus facilement transférable à l'adjectif déonomastique.

3. En effet, ces formations sont généralement envisagées du point de vue morphologique. Par ailleurs, le nom propre en général n'a été jusqu'à présent que partiellement abordé dans une perspective discursive (voir Lecolle, Paveau, Reboul-Touré, 2009). 


\section{Précisions terminologiques et critères constitutifs du corpus}

Conformément à l'hypothèse d'une parenté des statuts de l'emploi métaphorique du nom propre et de l'adjectif dérivé de celui-ci, nous emprunterons, dans notre analyse, un certain nombre de termes opératoires à la bibliographie, très vaste et issue de différentes approches, concernant l'usage du nom propre en discours. Notamment, nous puisons dans Jonasson (1994), Gary-Prieur (1994), Siblot-Leroy (2000), les termes référent originel pour indiquer le référent attitré du nom propre (Poutine, pour revenir à l'exemple 1); ceux de référent discursif pour désigner, en revanche, le référent-cible, c'est-à-dire l'entité à laquelle l'adjectif se rapporte parvoie figurale (Erdogan, en l'occurrence); et le terme contenu pour nommer la portion de traits sémantiques constituant le noyau d'« exemplarité» ou de «notoriété» du nom propre. Le contenu est donc une construction collective, à l'évidence essentielle pour rendre possible l'emploi métaphorique, à la fois du nom propre et de son dérivé. Suivant l'acception de Gary-Prieur4, il s'agit d'une entité intrinsèquement instable : issu d'un processus de sélection et de réduction, variable selon les sujets, les cultures, les époques, le contenu est soumis à une pression discursive particulièrement forte, les emplois figuraux fonctionnant toujours, comme on l'a souligné pour l'exemple 1, dans le cadre de l'allusion et, partant, de la co-énonciation5 maximale.

Pour construire le corpus des occurrences de berlusconien, nous avons consulté la base de données Factiva à partir de janvier 2010, ainsi que les archives électroniques de L'Express, L'Expansion, Libération, Le Nouvel Observateur, Le Point, Le soir.be, sans limitation de date. Dans l'ensemble pléthorique d'occurrences répondant à notre requête, nous avons été obligée de procéder à une sélection manuelle : les emplois relationnels de l'adjectif (ex:le gouvernement berlusconien $\rightarrow$ le gouvernement de Berlusconi), de loin les plus nombreux, ont été systématiquement écartés, ainsi que les qualificatifs (responsables d'une qualification endogène et non figurale, généralement paraphrasable au moyen d'une glose comparative : ex. un spread berlusconien $\rightarrow$ un spread comme celui de l'époque berlusconienne).

À l'issue de ce travail de tri, nous n'avons retenu qu'une centaine d'occurrences d'emplois métaphoriques de l'adjectif dérivé, parmi lesquelles:

3. Le leader Joschka Fischer est fragilisé et il sera confronté en 2002 à une campagne

4. Suivant Gary-Prieur (1994, p. 51), le contenu est «l'ensemble des propriétés attribuées au référent initial dans un univers de croyance ». Gary-Prieur préfère parler d' «interprétation métaphorique » plus que d' «emploi », ce qui ne nous empêche pas de puiser à sa terminologie.

5. Dans cet article, le terme «co-énonciation» n'est pas utilisé suivant l'acception de Rabatel (2005), mais indique plus largement l'opération de co-construction du sens qu'opère le destinataire, auquel nous réservons l'appellation, usuelle depuis Culioli, de co-énonciateur. 
électorale de type berlusconien, menée par un adversaire plus rude que prévu, le conservateur bavarois Edmund Stoiber. (L'Expansion, 21 juin 2001)

4. [Le discours de Toulon de Nicolas Sarkozy] était une farce, une gesticulation berlusconienne. (Le Nouvel Observateur, 12 janvier 2009)

À l'instar de l'exemple 1, et à l'opposé des emplois que nous avons exclus de notre corpus, la qualification réalisée par berlusconien dans les énoncés 3 et 4 est de nature exogène (référent originel $\neq$ référent discursif), un transfert figural se vérifiant entre le référent originel (Berlusconi, nom propre base du dérivé) et le référent discursif (représenté respectivement par Joschka Fisher dans l'exemple 3 et par Nicolas Sarkozy dans l'exemple 4). Le contenu du référent originel se rapportant au référent discursif comporte une liste indéfinie de possibilités attributives, le co-énonciateur émettant une série d'hypothèses puisées dans le noyau de notoriété du nom propre-source et orientées par le point de vue de l'énonciateur. Il apparaît, en effet, bien difficile de saisir toute la portée de la référenciation (Apothéloz, Reichler-Béguélin, 1995) qu’opère une expression telle qu' " une campagne électorale de type berlusconien », celle-ci pouvant être une campagne dépensière, basée sur une propagande menteuse, sur la manipulation des médias, etc. De manière encore plus évidente, il est pratiquement impossible de clore la liste des qualificatifs correspondant à « une gesticulation berlusconienne» : s'agit-il d'une gesticulation farcesque, tapageuse, clinquante, baratineuse, trompeuse, mystificatrice, etc. ? Outre la plasticité intrinsèque du contenu, les adjectifs utilisés dans les exemples 3 et 4, tout comme le nom propre en emploi métaphorique (exemple 2), mobilisent des cadres prédiscursifs propres et spécifiques à une collectivité (Paveau, 2006), qui peuvent se révéler plus ou moins contraignants, dans leur interaction avec le point de vue de l'énonciateur et l'interprétation du destinataire. Le caractère relatif et évolutif de leur fonctionnement, typiquement lié à une «praxis » langagière (Siblot, 1987, 1998), est largement confirmé dans une optique contrastive : l'utilisation de l'adjectif berlusconiano dans un corpus de presse italien n'amènerait pas du tout aux conclusions découlant de l'observation d'un corpus de presse français. À cet égard, bien que la comparaison interculturelle ne soit pas l'objet de notre étude, il est significatif de constater que l'emploi métaphorique (à la fois du nom propre Berlusconi et de son dérivé berlusconiano) est beaucoup plus rare en Italie qu'en France, la position différente assumée, dans les deux pays, par l'«objet mondain» Berlusconi déterminant à l'évidence des différences importantes dans la circulation de l' « objet discursif »6.

6. Sur la différence entre «objet mondain » et « objet discursif», nous renvoyons à Mondada, Dubois, 1995. 


\section{Analyse du corpus : les emplois métaphoriques de l'adjectif berlusconien}

Le discours de la presse française de ces dernières années porte de nombreuses traces d'opérations comparatives fondées sur l'objet discursif Berlusconi. Ces traces ont en général une fonction dépréciative, pouvant se présenter sous des formes diverses, qui vont des textes argumentés, posant l'analogie de façon explicite, jusqu'aux simples suggestions confiées à des déformations du signifiant, telles que l'amalgame Silvinique Berluskhan, ou les composés sarko-berlusconiens, déclinés sur le moule du "sarkoberlusconisme» (Musso, 2008). Dans ce cadre, un procédé fort commun est représenté par la mention directe du nom propre Berlusconi, réalisant des usages discursifs divers, depuis l'«emploi exemplaire »7 de l'exemple 5 jusqu'à l'emploi métaphorique de l'exemple 6, issu du même dispositif figural que l'énoncé 2 concernant l'objet discursif Poutine :

5. Mais il [Alexandros, un jeune Grec interviewé] se dit désormais «déçu» et "tenté dans une certaine mesure» par l'émergence d'une nouvelle personnalité, déconnectée des deux grands partis, «un Berlusconi à la grecque». (Libération, 9 décembre 2010)

6. À Sarkozy, on ne demande pas de comptes [...] : la brume s'épaissit un peu plus autour du Berlusconi hexagonal, jusqu'à lui faire l'aura d'un visionnaire... (Libération, 20 février 2007)

Suivant notre hypothèse, l'emploi métaphorique de l'adjectif berlusconien dépend de la circulation médiatique du nom propre dont il est issu et notamment de la configuration changeante de son contenu, ou noyau de notoriété. Au point de vue sémantico-énonciatif, le qualificatif berlusconien partage donc le statut flottant de l'insinuation, du clin d'œil ironique qui préside à la généralité de ces phénomènes de court-circuit dénominatif. Toutefois, le plan syntaxique sur lequel s'actualise la qualification mérite une analyse préalable, la prise en compte de ce niveau étant fondamentale pour saisir les enjeux du travail de négociation du sens auquel ce déonomastique nous convie. En effet, en tant qu'adjectif, les fonctions sémantiques qu'accomplit berlusconien sont déterminées par la position que cet élément occupe à l'intérieur du syntagme nominal d'appartenance, ainsi qu'au rôle que celui-ci joue dans l'entourage discursif. Malgré la difficulté à isoler les effets relatifs aux différents paliers, qui fonctionnent de façon cumulative, nous avons choisi, pour des raisons de clarté, de distinguer le niveau du co-texte immédiat et le niveau du co-texte

7. Pour une hypothèse de continuité entre un type d'emploi et l'autre, voir Leroy, 2005a. 
élargi, ou du contexte général 8 , essayant d'articuler des critères prioritairement syntaxiques et énonciatifs dans les deux sections qui suivent.

\section{Le co-texte immédiat}

\section{Position adjectivale et fonction syntaxique}

La position privilégiée de la qualification figurale berlusconien est celle de l'épithète postposée à un nom, comme cela se vérifie dans l'exemple 4 (une gesticulation berlusconienne) ou dans l'exemple 7 ci-dessous. Cet énoncé, dans lequel le nom auquel l'adjectif se réfère (dorénavant appelé nom recteur) est celui de la formation politique grecque «Aube dorée », montre le degré d'audace que peuvent atteindre les assimilations pivotant sur l'adjectif métaphorique :

7. L'Aube Dorée berlusconienne promet deux choses : une guerre pathétique contre l'Allemagne et une bataille autarcique contre l'euro. (L'Express, 27 juin 2012)

En effet, dans l'exemple 7, la qualification berlusconienne fait l'objet d'une présupposition discursive (Biglari, Bonhomme, à paraître), c'est-à-dire d'un élément allégué comme allant de soi, que l'énonciateur ne se donne pas la peine de justifier, une analogie explicite entre le parti néo-nazi grec et le berlusconisme italien étant bien plus difficilement proposable et soutenable.

En revanche, l'adjectif métaphorique peut parfois donner lieu à une véritable prédication assertive, l'épithète post-nominale berlusconien apparaissant dans un syntagme nominal attributif. Les occurrences de ce genre, moins fréquentes dans notre corpus, relèvent de préférence du discours rapporté (ou, pour mieux dire, représenté), comme on le voit dans l'exemple 8 :

8. Daniel Cohn-Bendit dénonce : «Le style de Sarkozy c'est un style très berlusconien. Cette histoire, par exemple, de mettre sur son facebook sa rencontre avec “Carla chouchou mama” tu vois moi j'ai fait ceci, j'ai vu le Premier irakien, ah c'est génial lui dit Carla », se moque-t-il. (Le Point, 24 mai 2009)

Par rapport à l'exemple 7 , l'énoncé 8 met en scène une prédication explicite. C'est sans doute à cause de cela que ce type de tournure figure de façon privilégiée dans des discours rapportés, la prise en charge énonciative ne revenant pas ainsi au journaliste, ou énonciateur premier, qui se borne à référer les propos assertés par un énonciateur second (Daniel Cohn-Bendit, en l'occurrence).

En outre, l'exemple 8 illustre une autre caractéristique de l'emploi métaphorique du déonomastique adjectival : sa compatibilité avec un opérateur de gradualisation (très, assez, etc.). La qualification gradualisée, qu'attestent par exemple les énoncés suivants, est fort fréquente :

8. Ces termes sont utilisés ici de la même manière que dans Bonhomme, 2005. Sur la relation des figures du discours et de leur contexte, voir Gaudin-Bordes, Salvan, 2015. 
9. Il (Moïse Katumbi) est flamboyant [...], c’est un vrai acteur ayant une générosité à la Mobutu. C'est un richissime homme d'affaires juif italien élu démocratiquement, donc hyperpopulaire. Homme d'affaires un peu berlusconien. (Le Soir.be, $1^{\text {er }}$ avril 2009)

10. Nicolas Sarkozy affiche une irrépressible fringale de pouvoir [...]. Monarque absolu [...] cloué au pilori pour l'exhibition de ses tumultes sentimentaux, ostracisé pour son style trop berlusconien [...] (Libération, 20 février 2008)

L'opérateur de gradualisation assume la fonction de modaliser l'amalgame, car il est susceptible d'atténuer sa hardiesse (exemple 9) ou de l'intensifier, contribuant à rendre plausibles les vives critiques formulées (exemple 10). La modalisation suggère ainsi l'existence d'une échelle dans la proximité avec le parangon catégoriel.

Par rapport à l'emploi postposé, la position antéposée de l'épithète berlusconien se révèle rarissime dans notre corpus. Dans les quelques cas où cette disposition est attestée, l'expression définie qui en résulte acquiert une claire valeur de présupposition d'existence (voir Paissa, à paraître), conférant à la qualification métaphorique la force d'une vérité indiscutable, voire d'un authentique "effet d'évidence» (Guilbert, 2007). Effectivement, la qualification berlusconien précédant le nom recteur est présentée, dans un énoncé tel que l'exemple 11 ci-dessous, comme une propriété inhérente à son référent, jouissant de la «naturalité artificielle »9 d'une sorte d' "épithète de nature »:

11. [...] Pas plus que les Français n’ont été invités à débattre du sort d'un patrimoine qui leur appartient et dont le berlusconien Sarkozy croit disposer à sa guise. (Le Nouvel Observateur, 14 décembre 2009)

La construction attributive $(N+$ être + adjectif $)$, illustrée par l'exemple 12, est également peu fréquente :

12. C'est inquiétant cette tentative de mise au pas, c'est Berlusconien, c'est antidémocratique, c'est profondément choquant. (Le Nouvel Observateur, 13 novembre 2009)

Ce commentaire, publié dans un blog du Nouvel Observateur, concerne la prise de position du député UMPÉric Raoult envers la lauréate du prix Goncourt 2009, Marie Ndiaye, qui avait exprimé des jugements critiques contre Nicolas Sarkozy. À propos de cet exemple, il convient aussi de remarquer qu'il s'agit de la seule occurrence de notre corpus où Berlusconien est écrit avec la majuscule, vestige du lien qui se maintient parfois entre l'adjectif déonomastique et le nom propre dont il est issu.

En règle générale, la préférence accordée à la construction épithétique, par rapport à la construction attributive de l'adjectif dérivé du nom propre en

9. C'est nous qui proposons cet oxymore, pour indiquer l'effet de «naturalité » imposé par le point de vue discursif. 
emploi métaphorique, représente une confirmation du fonctionnement allusif de ce dispositif rhétorique. En effet, sa finalité principale est de permettre une caractérisation suggestive, sémantiquement vague et tendanciellement implicite, susceptible de réaliser des rapprochements hasardeux, comme dans les exemples $7,9,11,12$, peu ou prou modalisés, voire atténués, comme dans les exemples 8, 9, 10 .

\section{Le rôle du nom recteur}

La valeur et l'orientation axiologique du syntagme nominal à épithète berlusconien dépendent fondamentalement du nom recteur et du rapport que celui-ci entretient avec le référent discursif. En ce sens, deux cas de figure différents peuvent se présenter : soit le nom recteur coïncide directement avec le référent discursif de la qualification métaphorique (c'est ce qui se passe dans l'exemple 7 pour «Aube dorée » et dans 11 pour «Sarkozy»), soit il ne coïncide pas avec le référent discursif. Dans ce cas, l'adjectif renvoie au référent discursif de façon décalée, à cause de l'interposition d'un autre substantif en tant que tête du syntagme nominal (il en est ainsi pour «gesticulation » dans l'exemple 4, pour «style » dans les exemples 8 et 10). Cette tête nominale peut alors être sémantiquement et axiologiquement neutre ou, à l'inverse, être marquée. Dans le premier cas, le nom est constitué par un item au sémantisme faible, n'ayant qu'une fonction de support adjectival et ne présentant aucune marque axiologique. C'est ce qui se vérifie justement pour «style » et pour tout un micro-paradigme d'unités lexicales peu informatives, comme «modèle», «allant », «profil », "côté », etc. Dans le deuxième cas, le nom est sémantiquement et axiologiquement marqué : la visée argumentative et le trait dysphorique de la qualification adjectivale se doublent alors de l'orientation péjorative du nom employé, comme le montre l'exemple 13 :

13. Au-delà de ces objectifs conjoncturels, les facteurs enfonçant le débat politique français dans les marais berlusconiens ne manquent pas. (Libération, 7 janvier 2010)

La portée axiologique négative que véhicule l'adjectif berlusconien est en effet redoublée, dans cet exemple-ci, par la nature, à son tour figurale et négativement chargée, du substantif «marais ». Appartiennent à ce paradigme plusieurs combinaisons, à forte portée dépréciative, telles que «dérive berlusconienne» (ex. 14), "fort relent berlusconien » (ex. 17), «cancer berlusconien» (ex. 22). Le cadre énonciatif de ces dénominations à degré péjoratif élevé est généralement celui du discours « représenté », la responsabilité de l'appréciation négative revenant ainsi entièrement à un énonciateur second, dont on rapporte les propos (respectivement les Verts et le PS, dans l'ex. 14):

14. Les Verts ont fait plus contemporain, situant Sarkozy «entre dérive berlusco- 
nienne et népotisme à la Poutine», tandis que le PS évoque une «reprise en main » de la télévision publique. (Libération, 27 juin 2008)

En outre, le nom recteur suivi de l'adjectif berlusconien peut parfois occuper la position prédicative et donner lieu ainsi à une véritable recatégorisation du référent discursif, comme cela arrive pour "superhéros», sorte de dénomination résomptive des appositions nominales se référant à Poutine dans l'exemple ci-dessous. Dans ce cas de figure, l'adjectif et le nom recatégorisant se renforcent mutuellement, réalisant une prédication complexe, à la forte portée ironique et polémique :

15. Il [Poutine] est archéologue, chasseur de tigres, plongeur, motard, pilote de formule 1, crooner... En d'autres termes, il est un "superhéros berlusconien », écrit Thomas Gomart. (Les Échos, 5 janvier 2012)

Ce mécanisme de recatégorisation nominale et de redoublement analogique peut aboutir à des dénominations particulièrement insultantes : que l'on considère, par exemple, le cas de la double caractérisation métaphorique que François Bayrou réserve à Nicolas Sarkozy, lors de la présidentielle de 2007, de « petit Duce berlusconien » (Le Soir.be, 27 avril 2002). Dans cette expression figurale complexe, le substantif «petit Duce» (comportant, en plus, la modalisation ironique «petit », comme dans l'exemple 2), opère déjà un renvoi à l'un des pires souvenirs de l'histoire italienne du xxe siècle, auquel s'ajoute la qualification berlusconien .

Pour terminer cette partie consacrée au co-texte immédiat, on peut encore souligner un certain penchant de l'adjectif métaphorique à figurer dans des cotextes énumératifs. L'objectif de la qualification adjectivale négative est accentué, dans ces cas-là, par l'effet d'accumulation. Dans l'exemple 16, dont le référent discursif est représenté par Nicolas Sarkozy, outre l'adjectif berlusconien, d'autres dérivés de noms propres, utilisés également en emploi métaphorique (bonapartiste, thatchérien, blairiste, bushiste), s'entassent en cascade :

16. Libéral certes, mais tendance bonapartiste. De droite, oui, mais ascendant bling-bling. Thatchérien et blairiste, berlusconien et bushiste. (Libération, 22 décembre 2007)

L'énumération, qu'on peut voir à l'œuvre aussi dans l'exemple 12, assemble pêle-mêle une suite d'étiquettes fort hétéroclites au point de vue politique, ce qui en dit long sur le degré d'approximation avec lequel ces qualifications métaphoriques sont exploitées dans le discours de presse.

\section{Le co-texte élargi et le contexte général}

Compte tenu des configurations sémantico-syntaxiques et énonciatives qui sont déterminées par le co-texte immédiat, on peut se pencher maintenant sur le cadre plus large de la qualification métaphorique et précisément sur les 
interactions que le couple référent originel / référent discursif établit avec le cotexte élargi et le contexte général, ainsi que sur la nature sémantique de l'élément déclencheur de l'analogie et de la cible argumentative.

\section{L’interaction référent originel-référent discursif et le co-texte élargi : présence/absence de la motivation de l'analogie}

La sélection des propriétés formant le contenu d'un adjectif métaphorique, susceptible d'être transféré par voie analogique à un référent discursif est, comme nous l'avons vu, le produit d'une opération de réduction dépendant du degré de notoriété acquis par le référent originel sur la scène médiatique. Malgré le caractère stéréotypé de la représentation collective dérivant de cette réduction, nous avons constaté qu'il n'est pas toujours aisé d'identifier les propriétés formant le contenu, puisqu'elles se présentent en faisceau et que leur portée polyphonique s'atteste à des degrés variables (la liste des calculs interprétatifs demeurant ouverte, comme nous l'avons vu ci-dessus pour les exemples 3 et 4).

À cet égard, le co-texte élargi peut présenter des éléments susceptibles d'aiguiller l'interprétation, dans la mesure où ils proposent une justification de la qualification métaphorique. Dans les deux exemples ci-dessous, la motivation co-textuelle de l'analogie est explicitée par les segments que nous avons soulignés (en italiques) :

17. Le porte-parole du PCF, Olivier Dartignolles, a fustigé une "présidence totalement décomplexée au fort relent berlusconien » qui «se fixe comme objectif [...] d'anesthésier l'opinion publique en contrôlant les grands moyens d'information ». (L'Expansion, 22 mai 2007)

18. Pim Fortuyn a en lui quelque chose d'un Bruno Mégret. Ne rêve-t-il pas d'un front des droites dressé contre l'establishment? Il est assez proche de Haider [...]. Il y a enfin dans sa dynamique un allant berlusconien, un goût du toc et du brillant. (Le soir.be, 8 mars 2002)

L'exemple 17 se réfère une fois de plus à Nicolas Sarkozy : le jugement dépréciatif redoublé (voir ci-dessus, «Le rôle du nom recteur»), issu de l'association du qualificatif berlusconien appliqué à un nom recteur déjà péjoratif (relent), revient au porte-parole du PCF, dont l'énonciateur premier choisit de rapporter également l'argument motivant l'amalgame proposé (l'« objectif d'anesthésier l'opinion publique » par le contrôle des « grands moyens d'information » représentant, selon le point de vue de l'instance énonciative seconde, une visée commune à Nicolas Sarkozy et à Silvio Berlusconi). L'exemple 18 présente, quant à lui, une description de Pim Fortuyn. La responsabilité de l'opinion exprimée est, cette fois-ci, entièrement endossée par le journaliste, Pascal Martin : après avoir mobilisé, pour définir l'homme politique néerlandais, des références à Bruno Mégret et à Haider, le rédacteur de l'article souligne l'« allant berlusconien » de Pim Fortuyn, le motivant par son « goût du toc et du brillant». 
Dans d'autres cas, la qualification métaphorique n'entraîne la présence d'aucun élément explicite déterminant l'analogie. À ce propos, nous avons pu constater que l'élément justificatif s'avère plus implicite lorsque le locuteur peut compter sur un savoir largement partagé, comme le montre l'exemple 19, où il est question d'une comparaison entre Carla Bruni Sarkozy et MarieAntoinette. Dans cet énoncé, le qualificatif berlusconien peut bien se passer d'explications, tant le prédiscours et l'interdiscours sur les frasques sentimentales de Silvio Berlusconi sont universellement connus :

19. L'«Autrichienne» et l'Italienne; deux femmes jeunes, jolies et «décoratives»; [...] un mariage pour cimenter l'alliance des Bourbons et des Habsbourg là, des Sarkozy et du storytelling berlusconien ici. (L'Express, 26 mars 2012)

Bien qu'une correspondance entre Silvio Berlusconi et Nicolas Sarkozy, pour ce qui est des rapports avec l'autre sexe, soit assez discutable (d'un côté il y a un lien conjugal et, de l'autre, une virilité affichée tous azimuts), on comprend aisément ce que le journaliste entend indiquer par l'expression « un storytelling berlusconien », les vicissitudes du couple présidentiel français ayant été entourées, de 2008 à 2012, d'un ensemble de rumeurs médiatiques comparables à celui accompagnant la chaotique vie amoureuse de Silvio Berlusconi.

L'observation du co-texte élargi confirme, en règle générale, l'existence d'une solidarité entre l'ampleur de l'interdiscours et des savoirs partagés et la présence/absence de motivations affichées pour soutenir l'analogie, l'ouverture à la co-énonciation s'avérant variable selon l'articulation de ces éléments. Cependant, les situations ne manquent pas où le co-texte n'offre guère d'éléments justifiant la qualification métaphorique, le potentiel interprétatif s'élargissant alors au maximum.

\section{La nature du référent discursif : ethos personnel, événement, contexte général}

Comme on peut le constater dans plusieurs exemples cités jusqu'ici, l'analogie que suppose l'adjectif métaphorique peut toucher soit l'ethos du référent discursif (le style, l'allant, le storytelling, etc.), soit un événement précis, soit encore un ensemble hétérogène, relevant du cadre sociopolitique général. Si c'est l'ethos du personnage qui est le plus fréquemment concerné par la caractérisation métaphorique berlusconien, comme le montrent de nombreux exemples (ex. 8, 9, 10,15, 18, 19, etc.), le contenu de l'adjectif peut présenter aussi un enracinement événementiel. Par exemple, toute la question de l'expulsion des Roms de la part du «clan sarkozyen » en 2010 a été à l'origine d'une surenchère de comparaisons avec la ligne politique berlusconienne :

20. À cet héritage (des valeurs de la Révolution de 1789), le clan sarkozyen préfèret-il le modèle berlusconien en cours de l'autre côté des Alpes, où les Roms sont traqués et pourchassés, livrés en pâture aux nostalgiques du fascisme et aux agités de la Padanie? (L'Humanité, 30 juillet 2010) 
En outre, la nature du référent discursif peut relever de différentes catégories. Dans la plupart des cas, il s'agit d'un autre personnage appartenant à l'actualité médiatique (comme nous l'avons vu, la cible première, dans la presse française, est constituée par Nicolas Sarkozy, mais nous avons repéré également Vladimir Poutine, Moïse Katumbi, Pim Fortuyn, Victor Orbàn, et même le très jeune prince Harry). Cependant, le transfert analogique peut aussi déborder du cadre strict de la personne et s'élargir à un cadre situationnel plus complexe, à l'instar de l'exemple 13 ci-dessus, concernant le débat politique français, menacé de s'enliser dans les «marais berlusconiens». De cette manière, le «berlusconisme » et la «berlusconisation $»^{10}$ se posent comme des catégories politiques à vocation universelle. Là aussi, l'élément déclencheur de l'analogie peut être de nature événementielle, comme dans l'exemple 21 ci-dessous, qui remonte à 1989 et concerne le refus d'une réglementation des chaînes de télévision. Grâce au qualificatif berlusconien, ce déni compare l'attitude de l'ACT au régime télévisuel italien, marqué depuis les années quatre-vingt par le privilège dont bénéficient les entreprises de communication appartenant au «Cavaliere » :

21. La guerre des chaînes bat son plein en France. [...] L’ACT (Association des télévisions commerciales européennes) est passée à l'acte en déclarant un «non » berlusconien aux quotas et réglementations européennes contraignantes. (Le soir.be, 16 octobre 1989)

Cette qualification élargie à la globalité d'une position ou d'une perspective politique apparaît volontiers dans des syntagmes nominaux définis, doués par là d'une indiscutable présupposition d'existence. La qualification qu'effectue l'adjectif métaphorique berlusconien prend facilement, dans les occurrences de ce type, une allure de prophétie sinistre et apodictique, comme le montrent les exemples 22 et 23 :

22. Au moment où notre pays va devoir se doter d'un arsenal législatif adapté aux nouvelles technologies, il faut méditer sur le «cancer» berlusconien. (Le Point, 17 juin 1995)

23. Prenons garde : si l'Italie reste bien notre laboratoire, le rêve berlusconien deviendra bientôt le cauchemar des Européens. (L'Expansion, 1 juin 2006)

Ces énoncés illustrent une opération de qualification dans laquelle la référence à Silvio Berlusconi, comme on l'a vu plus haut, se double de la connotation péjorative de la tête nominale du syntagme nominal. Dans l'exemple 22, le système des télécommunications italien constitue le «cancer» berlusconien sur lequel les autres pays, et notamment la France, ont intérêt à « méditer», l'orientation axiologique négative de la métaphore «cancer» étant à peine modalisée par des guillemets de distanciation, formant «îlot textuel» (Authier-

10. Voir Paissa, Druetta, 2013 pour quelques considérations sur d'autres dérivés du nom propre Berlusconi. 
Revuz, 1996). Dans l'exemple 23, le «rêve berlusconien » renvoie au thème général de l'article, traitant de l'aspect le plus inquiétant du «phénomène Berlusconi », c'est-à-dire la concentration, dans une grande démocratie européenne, du pouvoir médiatique et politique sous la même houlette : c'est en ce sens que le «rêve » d'emprise politique sur l'information pourrait devenir «le cauchemar des Européens». Or, si la visée de la qualification figurale, portant dans ces deux exemples sur la collusion berlusconienne entre pouvoir médiatique et politique, apparaît suffisamment claire, les circonstances situationnelles de l'opération de référenciation qu'effectuent les syntagmes nominaux demeurent assez floues, permettant des calculs interprétatifs importants. En effet, au vague des substantifs métaphoriques, auxquels l'adjectif berlusconien se réfère («cancer», « rêve »), s'ajoute l'imprécision des déictiques, ainsi que l'estompage du cadre temporel, la perspective d'un temps indéfini caractérisant les deux énoncés (dans 22, une époque s'ouvre où «il faut méditer»; dans 23, le moment arrivera où le «rêve » "deviendra » un «cauchemar»).

Enfin, dans d'autres occurrences, l'assignation figurale de la qualité berlusconien peut s'agrandir ultérieurement, intéressant un contexte de plus en plus large et indéterminé : celui-ci peut aller, grâce à l'abstraction du nom recteur, jusqu'à l'évocation d'une entité moralement et politiquement menaçante, représentée par « l'éternel berlusconien qui concerne l'Italie et le monde entier» (Courrier international, 1 juin 2011). La portée contextuelle d'un énoncé de ce type est évidemment fort vaste : l'ouverture à la co-énonciation y atteint donc le degré le plus élevé.

Suivant l'hypothèse d'une affinité de fonctionnement entre l'emploi métaphorique du nom propre et de l'adjectif dérivé de celui-ci, nous avons décrit le comportement sémantico-discursif du qualificatif berlusconien, dans des dispositions co-textuelles et contextuelles diverses. L'observation du corpus confirme notre hypothèse de départ : un qualificatif comme berlusconien assume dans le discours la même fonction résomptive, allusive et ironique que joue l'emploi métaphorique du nom propre (Leroy, 2005b). En conclusion de notre analyse, nous pouvons ajouter que les amalgames issus de l'adjectif dérivé du nom propre semblent jouir d'une liberté et d'une facilité de circulation encore plus fortes que celles dont jouit le nom propre dans son emploi métaphorique. Alors que celui-ci est généralement soumis au « principe de la continuité ontologique » (Gary-Prieur, 1994, 2009 ; Leroy, 2005b), imposant des restrictions catégorielles (un nom propre de personne ne se réfère en principe, par voie figurale, qu'à une autre personne, un nom propre d'évènement s'applique en général à un autre évènement, et ainsi de suite), la qualification adjectivale, comme nous l'avons vu, peut s'étendre à des référents dont les contours sont à leur tour figuraux et imprécis (le marais, le climat, l'éternel, etc.). Déjà floue et ambigüe en elle-même, la caractérisation s'appuyant sur l'adjectif métaphorique s'avère donc particulièrement malléable car, jouant sur l'entourage 
phrastique et discursif, elle peut encore sensiblement élargir son flou catégoriel et sa capacité de référenciation. En conséquence de cette plasticité, le déonomastique adjectival présente des possibilités d'actualisation discursive encore plus amples que le nom propre. En même temps, il apparaît moins aisément réfutable : par rapport à la mention directe du nom propre, l'adjectif déonomastique fait figure de mot beaucoup plus routinier et anodin, à cause sans doute de sa nature de mot dérivé, lui conférant un statut d'unité lexicale plus motivée et moins arbitraire. À juste titre, Marie-Noëlle Gary-Prieur (1994, p. 91) remarquait que «ton gaullien » présente un lien avec le référent originel sensiblement plus relâché que «ton de de Gaulle». Or, c'est justement par son éloignement plus grand du référent originel que le dérivé adjectival est plus couramment reçu et, par conséquent, moins facilement désavoué.

Tout en expliquant la grande productivité néologique des dérivés du nom propre et leur présence massive dans la communication médiatique, ces observations ouvrent la voie à des considérations portant sur les enjeux véritables de ce genre d'opérations discursives.

Sémantiquement vague et foncièrement dialogique, le dérivé du nom propre en emploi métaphorique sollicite l'adhésion du co-énonciateurà un tour de force discursif, à une opération de placage fondée sur une analogie qu'on assène, dans la plupart des cas, comme un facteur allant de soi, jouissant d'une présupposition d'existence incontestable, alors qu'il est de nature évidemment idéologique (Reboul, 1980). En effet, le vecteur analogique qu'on mobilise dépend de l'interaction d'éléments relatifs et contingents, comme l'orientation axiologique du point de vue présidant à l'énonciation et le contenu, la «notoriété» du nom propre, variable selon la configuration de la doxa interdiscursive à un moment donné. Le phénomène observé s’inscrit donc dans un questionnement plus général, touchant au véritable rôle que joue l'analogie dans le discours médiatique. Effectivement, dans cette pratique langagière, la fonction du relai de type analogique est souvent moins de révéler des ressemblances factuelles et démontrables que de créer, suggérer, voire prescrire des assimilations abusives, dont le caractère provisoire et éphémère n'estompe pas le fâcheux pouvoir de conditionnement, voire d'aveuglement des esprits. Par sa facilité, le mécanisme de l'équivalence simplificatrice et trompeuse prend ainsi volontiers la place de l'argumentation explicite, qui demande un travail beaucoup plus laborieux et patient, mais aussi plus respectueux des positionnements, des opinions et des perspectives différents. Des procédés extrêmement schématiques et réducteurs, tels que les emplois figuraux du nom propre et de ses dérivés, s’imposent donc aisément, bien qu'ils ne représentent, en définitive, que des tours fallacieux du langage, des forçages discursifs, destinés à servir les besoins d'immédiateté du langage médiatique. Par ailleurs, ces amalgames dénominatifs constituent, à notre avis, l'un des indices de la dérive vers la personnalisation et la spectacularisation du débat politique : faute de contenus et d'arguments originaux, 
celui-ci a souvent recours à la caricature verbale, alors que la dramatique complexité du monde exigerait, à l'inverse, plus de lucidité et une plus grande capacité à effectuer des distinguos subtils. Le statut à la fois paralogique, fallacieux et idéologique de ce genre de raccourcis expressifs ne va donc pas sans poser un problème d'éthique langagière, aussi bien aux professionnels de la communication qu'aux chercheurs en sciences du langage ${ }^{11}$.

\section{Références}

Apothéloz Denis, REICHLER-BÉguÉlin Marie-José, 1995, «Construction de la référence et stratégies de la désignation», Tranel : Travaux neuchâtelois de linguistique, no23, p. 227-271.

Authier-Revuz Jacqueline, 1996, «Remarques sur la catégorie de l'“îlot textuel” », Cahiers du français contemporain, n³ 3 , p. 91-115.

BARTNING Inge, NoAilly Michèle, 1993, "Du relationnel au qualificatif : flux et reflux», L'Information grammaticale, $\mathrm{n}^{\circ} 58$, p. 27-32.

BIGLARI Amir, Bonhomme Marc éd., à paraître, La présupposition entre théorisation et mise en discours.

Bon homme Marc, 2005, Pragmatique des figures du discours, Paris, Champion.

FLAux Nelly, 1991, "L'antonomase du nom propre ou la mémoire du référent », Langue française, no 92 , p. 26-45.

- 2000, "Nouvelles remarques sur l'antonomase», Les noms propres. Nature et détermination, D. Van de Velde, N. Flaux, W. De Mulder éd., Lille, Presses universitaires du Septentrion, p.117-146.

Gaudin-Bordes Lucile, Salvan Geneviève, 2015, Pratiques, n⿳0165-166, Étudier les figures en contexte. Quels enjeux? http://pratiques.revues.org/2374.

GARY-PRIEUR Marie-Noëlle, 1994, Grammaire du nom propre, Paris, PUF.

- 2009, "Le Nom propre, entre langue et discours», Le nom propre en discours, M. Lecolle et al. éd., Paris, Presses Sorbonne Nouvelle, p.153-168.

GUILBERT Thierry, 2007, Le discours idéologique ou la force de l'évidence, Paris, L'Harmattan.

JONASSON Kerstin, 1991, «Les noms propres métaphoriques. Construction et interprétation », Langue française, $n^{\circ} 92$, p. 64-81.

- 1994, Le Nom propre. Constructions et interprétations, Louvain-la-Neuve, Duculot.

KLEIBER Georges, 1994, "Y a-t-il de la métaphore sous les noms propres en antonomase? », Studia Romanica Posnianiensia, n 19, p. 37-52.

- 1995, "Sur la définition des noms propres, une dizaine d'années après", Nom propre et nomination, M. Noailly éd., Paris, Klincksieck, p. 11-36.

KOREN Roselyne, 2008, "Pour une éthique du discours. Prise de position et rationalité axiologique », Argumentation et Analyse du discours, nº 1/2008, L'analyse du discours au prisme de l'argumentation, R. Amossy, R. Koren éd., http://aad.revues.org/263.

11. Voir Koren, 2008, 2012. 
- 2012, "Langage et justification implicite de la violence. Le cas de l'“amalgame” ", Victor Klemperer. Repenser le langage totalitaire, L. Aubry, B. Turpin éd., Paris, Presses du CNRS, p. 93-105.

Lecolle Michelle, Paveau Marie-Anne, Reboul-Touré Sandrine éd., 2009, Le nom propre en discours, Paris, Presses Sorbonne Nouvelle.

LEROY Sarah, 2000, "Quels fonctionnements discursifs pour l'antonomase du nom propre?», Cahiers de praxématique, n035, p. 87-113.

- 2004, "L'antonomase du nom propre est-elle le lieu d'une médiation? », La Médiation. Marquages en langue et en discours, Rouen, Presses de l'Université de Rouen.

- 2005a, «L'emploi exemplaire, un premier pas vers la métaphorisation?», Langue française, $\mathrm{n}^{0} 146, \mathrm{p} .84-98$.

- 2005b, De l'identification à la catégorisation. L'Antonomase du nom propre en français, Louvain/Paris, Peeters.

LIGNON Stéphanie, 2002, "L'adjectif en -ien comme révélateur de phénomènes de concurrence ", BULAG, n²7, p. 135-150.

MoIRAND Sophie, 2007, Les discours de la presse quotidienne, Paris, PUF.

MondAdA Lorenza, DuBoIs Danièle, 1995, «Construction des objets de discours et catégorisation. Une approche des processus de référenciation», Tranel. Travaux neuchâtelois de linguistique, $\mathrm{n}^{0} 23$, p. 273-302.

Musso Pierre, 2008, Le sarkoberlusconisme, Paris, La Tour d'Aigues, L’Aube.

Paissa Paola, DruetTA Ruggero, 2013, «De Berlusconi à ... berlusconiesque. Polymorphies du nom propre dans le discours médiatique français», Linguistica applicata con stile. In traccia di Bice Mortara Garavelli, F. Geymonat éd., Alessandria, Edizioni dell'Orso, p. 105-127.

- à paraître, "Majorité silencieuse. Une formule à fondement présuppositionnel dans les campagnes présidentielles françaises de 2007 et 2012 », La présupposition entre théorisation et mise en discours, A. Biglari, M. Bonhomme éd.

Paveau Marie-Anne, 2006, Les prédiscours. Sens, mémoire, cognition, Paris, Presses Sorbonne Nouvelle.

RABATEL Alain, 2005, «La part de l'énonciateur dans la co-construction interactionnelle des points de vue », Marges linguistiques, n 9 , p.115-136.

Reboul Olivier, 1980, Langage et idéologie, Paris, PUF.

SAMOUTH Églantine, 2010, «L'adjectif bolivariano dans la presse vénézuélienne. Entre langue et positionnements politiques», Mots. Les langages du politique, n 93 , p. 47-63.

SiBlot Paul, 1987, «De la signifiance du nom propre», Cahiers de praxématique, n॰8, p. $97-114$.

- 1998, "Signifiance du praxème nominal», L'Information grammaticale, nº 77 , p. $24-27$.

Siblot Paul, LeroY Sarah, 2000, «L'antonomase entre nom propre et catégorie nominale », Mots. Les langages du politique, no 63, p. 89-104. 